\title{
Magnetic characteristics and AC losses of DC Type-II superconductors under oscillating magnetic fields
}

\author{
B. C. Robert ${ }^{1}$, and H. S. Ruiz ${ }^{1}$ \\ ${ }^{1}$ Department of Engineering and Leicester Institute for Space \& Earth \\ Observation Science of the University of Leicester, Leicester, LE17RH, U.K.
}

(Dated: January 17, 2018)

\begin{abstract}
Remarkable features on the magnetic moment of type-II superconducting wires of cylindrical shape, subjected to direct current conditions (DC) and transverse oscillating (AC) magnetic fields, are reported. We show how for relatively low amplitudes of the applied magnetic field, $B_{a}$, the superconducting wire rapidly develops a saturation state, $\left|M_{p}\right|$, characterizing the limits of magnetization loops that exhibit a Boolean-like behaviour. Regardless of the premagnetization state of the superconducting wire, we show how after two cycles of magnetic relaxation, boolean-like $\pm M_{p}$ states can be measured during the entire period of time from which the external magnetic field $B_{0}$ ranges from 0 to $\pm B_{a}$, with the signs rule defined by the sign of the slope $\Delta B_{0 y}(t)$. In addition, for the practical implementation of superconducting DC wires sharing the right of way with AC lines, we report that for relatively low values of magnetic field, $B_{a} \leq B_{P} / 2$, being $B_{P}$ the analytical value for the full penetration field in absence of transport current, $I_{t r}$, the use of semi-analytical approaches for the calculation of AC-losses leads to a significant underestimation of the actual contribution of the induction losses. This phenomena is particularly relevant at dimensionless fields $b_{a}<1-i_{a}^{2 / 3}$, being $b_{a}=B_{a} / B_{P}$ and, $i_{a}=I_{a} / I c$ the amplitude of an $\mathrm{AC}$ or DC transport current, due to the local motion of flux front profiles being dominated by the occurrence of transport current. On the other hand, we have found that regardless of the nature of the transport current, either be DC or $\mathrm{AC}$, when a transverse oscillating magnetic field greater than the classical limit $b_{a}=\left(1-i_{a}\right)$ is applied to the SC wire, the difference between the obtained AC losses in both situations results to be negligible indistinctly of the approach used, semi-analytical or numerical. Thus, the actual limits from which the estimation of the AC-losses can be used as an asset for the deployment of DC SC wires sharing the right of way with AC lines, against the sole use of $\mathrm{SC}$ wires for the transmission of AC transport current, are established.
\end{abstract}

\section{INTRODUCTION}

In densely populated urban areas, the deployment of new power lines implies the need to accommodate them into existing duct banks, aiming to reduce the overall costs of cable installation, surpass the conflict with the lack of construction space, and overcome the severe restrictions that right of way policies might imply [1, 2]. Due to the reduced use of space by state of the art superconducting cables, their high transport current capabilities [3], resilience properties against fault conditions 4], and nearly-zero resistive losses under DC conditions, type-II superconductors are expected to be a game changer in the designing of the next generation of $\mathrm{DC}$ power networks. However, the transition of power distribution grids from a dominantly AC network to a DC network advocates for the sharing of the right of way, as this could be the most practical way to reduce the development costs associated to the production and installation of SC cables with demanding time frames for operation.

Nevertheless, the understanding of the electromagnetic response of DC SC wires subjected to transverse AC magnetic fields becomes remarkably tangled, as the added external magnetic field magnetizes the wire with power

* Electronic address: bcr4@leicester.ac.uk losses increasing after each cycle, being impossible to predict its behaviour from analytical methods. In this paper, we present a comprehensive study on the physical phenomena underlying the effects of applying a transverse AC magnetic field to a rounded type-II DC SC wire, from which we have found a compelling Boolean-like behaviour in the magnetic moment of the wire under a wide range of intensities of applied magnetic field and transport current. The main advantage of using this geometry for the numerical modelling of type-II superconductors, relies on the possibility to compare the numerical results with exact or semi-analytical approaches, what allows a direct grasp of the physics involved whilst a phenomenological benchmark for the implementation of practical applications is being developed. In fact, the simple notion of this $2 \mathrm{D}$ geometry, i.e., by assuming that the length of the $\mathrm{SC}$ wire is much greater than its radius, and the critical state theory for the macroscopic modelling of the electromagnetic properties of type-II SCs [5] 8], has allowed to predict and explain multitude of experimental phenomena including but not limited to, the magnetization and demagnetization of SCs under crossed and rotating magnetic field experiments 913 , the low pass filtering effect in the magnetic moment of AC SC wires [14, and strong patterns of localization of the density of power losses inside the SC materials under magnetic and electrical stress conditions [15, 16].

In this paper, the electromagnetic problem is solved 

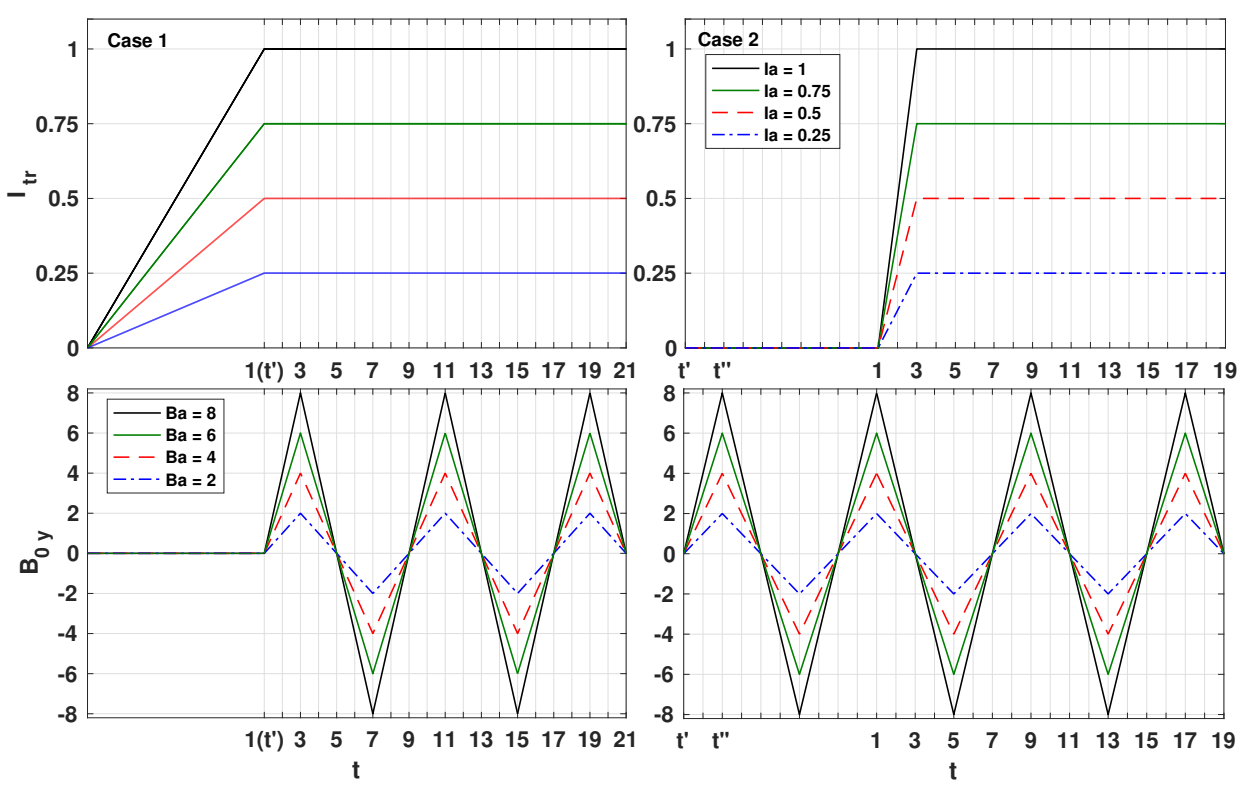

Figure 1. (Color online) Sketch of the analysed experimental processes where a rounded type-II SC wire of radius $R$, is subjected to the simultaneous action of an external AC magnetic field, $B_{0 y}(t)$, and a DC transport current $I_{t r}(t)$, of amplitudes $B_{a}$ and $I_{a}$, respectively. Case 1 (left pane) represents the situation when the DC current is applied to a magnetically virgin SC sample, whilst the analogous situation for a premagnetized sample is illustrated in case 2 (right pane). For the sake of simplicity, the magnetic field is presented in units of $\left(\mu_{0} / 4 \pi\right) J_{c} R$, and $I_{t r}$ is given in units of $I_{c}$.

via the variational optimization method for critical states problems developed by Bad'ia, López and Ruiz [6, 7], which for the case of infinitely long SC samples of finite cross section $\Omega$, is reduced to the minimization of the integral functional

$$
\begin{aligned}
& \mathcal{F}\left\{\mathbf{J}_{\mathrm{t}+1}\right\}=\frac{8 \pi}{\mu_{0}} \int_{\Omega}\left(\Delta \mathbf{B}_{0}\left(\mathbf{r}_{i}\right) \times \mathbf{r}_{i}\right) \cdot \mathbf{J}_{\mathrm{t}+1}\left(\mathbf{r}_{i}\right) d^{2} \mathbf{r} \\
& +\frac{\mu_{0}}{8 \pi} \int_{\Omega} \int_{\Omega^{\prime}} M_{i j} \mathbf{J}_{\mathrm{t}+1}\left(\mathbf{r}_{i \in \Omega}\right) \cdot \mathbf{J}_{\mathrm{t}+1}\left(\mathbf{r}_{j \in \Omega^{\prime}}\right) d^{2} \mathbf{r} d^{2} \mathbf{r}^{\prime} \\
& -\frac{\mu_{0}}{4 \pi} \int_{\Omega} \int_{\Omega^{\prime}} M_{i j} \mathbf{J}_{\mathrm{t}}\left(\mathbf{r}_{i \in \Omega}\right) \cdot \mathbf{J}_{\mathrm{t}+1}\left(\mathbf{r}_{j \in \Omega^{\prime}}\right) d^{2} \mathbf{r} d^{2} \mathbf{r}^{\prime}
\end{aligned}
$$

with the inductance matrices defined as $M_{i j}=1 / 2$ for all $\mathbf{r}_{i}=\mathbf{r}_{j}$, and $M_{i j}=\ln \left(\mathbf{r}_{i j}\right)$ for all $\mathbf{r}_{i} \neq \mathbf{r}_{j}$, being $\mathbf{r}_{i j}$ the distance between two elements of current located at two different positions inside the SC sample. The time dependence of the electromagnetic sources is controlled between two successive time steps $t<t+1$, on the one hand, by the first term of the minimization functional where $\Delta \mathbf{B}_{0}\left(\mathbf{r}_{i}\right)$ defines the rate of change of the applied magnetic field, $\mathbf{B}_{0}$, and on the other hand, by the condition of transport current " $I_{t r}(t)$ " which implies to add the mathematical constraints

$$
\int \mathbf{J}_{\mathrm{t}}\left(\mathbf{r}_{i}\right) d^{2} \mathbf{r}=I_{t r}(t) \leq I_{c} .
$$

The global minimization of the system of Eqs. 1 \& 2 which gives place to an accurate determination of the local profiles of current density (Section II), is commonly known as the integral formulation of the critical state model or $J$-formulation [15, 17, which is equivalent to the the solution of the PDE system of Faraday and Ampère's laws into the nowadays most popular $H$-formulation [18. The latter can be easily implemented in commercial computational packages such as COMSOL Multiphysics 911, but under the expense of having to pay a proprietary license and furthermore, having acquired enough experience on the numerical modelling of type II superconductors to be able to ensure that the obtained solution does not get anchored to a local minimum during the iterative solution of a two-point boundary value problem without well-defined extreme values, as with exception of problems possessing strongly convex properties [19, 20], global solutions of PDE systems are not always verifiable, given that the metrics for the magnetic field $\mathbf{B}$ cannot be homogeneously defined for the entire space 21. Nevertheless, within the optimal functional developed at Eq. 1, the metrics of the control variable $\mathbf{J}$, i.e., the current density, is explicitly defined by the critical state law $\left[6, \mathbf{J} \leq \mathbf{J}_{c}\right.$, and additionally, by the constraint for the transport current condition (Eq.22). In our case, for the computational minimization process a free software licence called Lancelot has been used which has the advantage of automatically adjusts the penalty parameter and Lagrange multiplier estimates, ensuring the convergence of the constrained optimization problem to a global solution, by implementing the trust-region approach or convex region into the quadratic model of the augmented Lagrangian function or so-called optimization function (Eq. 1), and within the constraints that charac- 
terize the physics behind the control variable. Thereafter an improvement to the quadratic model is sought using either a direct-matrix or truncated conjugate-gradient algorithm, systematically changing the size of the trusted region, such that the propagation of local minima is avoided. For more technical details on the capabilities of Lancelot, and how problems such as the one stated in this paper can be solved, we advise to the reader the Refs. 22, 23].

Then, in Section III the magnetic moment (per unit length) of the superconducting wire is calculated by numerical integration of the minimized distribution of profiles of current, i.e., as

$$
\mathbf{M}(t)=\frac{1}{2} \int_{\Omega} \mathbf{r}_{i} \times \mathbf{J}_{\mathrm{t}}\left(\mathbf{r}_{i}\right) d^{2} \mathbf{r},
$$

and to complete this study, the hysteretic losses per unit volume $(\Phi)$ and time, and for cyclic excitations of frequency $\omega$, i.e., the so-called AC-losses,

$$
L=\omega \oint_{h . c .} d t \int_{\Phi} \mathbf{E} \cdot \mathbf{J} d \Phi .
$$

can be calculated by the closed integral of the specific power density of energy losses $\mathbf{E} \cdot \mathbf{J}$ after the period elapsed for the magnetic relaxation of the profiles of current density, i.e., for the hysteretic cycle (h.c) which has to be determined by inspection of the numerical results for the profiles of current density or the symmetry of the magnetization curves.

In brief, this paper is organised as follows. In Section III. the local dynamics of the flux front profile of current density and the overall dynamics of the magnetic flux lines as a function of time, are both presented for different conditions of DC current, $I_{t r}$, and applied AC magnetic field, $B_{0 y}$. The influence of the DC current on virgin and premagnetized samples is also studied. Then, in Section III the most relevant characteristics on the magnetization profiles of the SC wire, together with their connection with the calculation of the AC-losses and the identification of Boolean-like magnetic moments are discussed in detail. Finally, Section IV is devoted to summarize the main conclusions of this work.

\section{DYNAMICS OF FLUX FRONT PROFILES}

In terms of the direction of the magnetic field created by a two dimensional SC wire, e.g., a wire of cylindrical cross section with radius much smaller than its length, the electromagnetic problem can be reduced to a $1 \mathrm{D}$ model with exact analytical solution, as long as the SC wire is exposed either to a parallel magnetic field or a longitudinal transport current, but not both of them being applied at the same time [5, 8, Even in the case when the external magnetic field is applied transverse to the surface of the SC sample [11, 12, 24, 27, resulting in a 2D model accounting for the possible directions of the magnetic field created by induced magnetization currents, it is still not possible to conceive an exact analytical solution [10, 25, 28]. Nevertheless, under the base of knowing the shape of the flux front profile of current density inside the SC material, several semi-analytical approaches have been implemented in the past, these being able to calculate the magnetization and AC-losses characteristics of SC wires under certain conditions of applied magnetic field and transverse current 29, 30, although severe limitations of these approaches have been discovered by robust numerical methods with high accuracy levels [17, 25, 27, 31 34. Thus, in an attempt to mimic the conditions that an ideal SC wire sharing the right of way with another power cable might impose, and how these relate to the most popular semi-analytical approaches, in this section we report a simplified analysis for the intricate dynamics of flux front profiles of current density inside a DC SC wire of rounded cross section, that is simultaneously exposed to transverse AC magnetic fields of different amplitudes.

Before applying the condition of transport current, two different cases can be envisaged in terms of the magnetization state of the SC wire (see figure 1). The first case refers to a magnetically virgin SC wire where $I_{t r}$ is being applied until reaching its maximum DC value $\left(I_{a}\right)$, it before the external $\mathrm{AC}$ magnetic field is triggered on. In the second case, the $\mathrm{SC}$ wire is assumed to be premagnetized by the existence of an external $\mathrm{AC}$ magnetic field, it before injecting the DC transport current. For simulating the latter condition, we have assumed that the $\mathrm{SC}$ wire is firstly subjected to an AC magnetic field of certain amplitude, $B_{a}$, and once a full magnetic hysteresis cycle is achieved, the ramp of $I_{t r}$ is turned on until the $I_{a}$ value is reached. Then, a new hysteresis behaviour starts, covering the concomitant action of the AC field and the DC current regardless of the initial magnetization of the SC wire.

For case 1, the flux front profiles of current density $J_{i}= \pm J_{c}$ before $t^{\prime}$, i.e., the time step when $B_{0 y}$ is activated, show the classical concentric behaviour with elements of current occurring from the sample's surface towards its geometrical centre as $I_{t r}$ increases. The resulting flux front profile of current density at this time step, ' 1 ', for a maximum transport current $I_{a}=0.5 I_{c}$ is displayed in the first subplot of figure 2. Then, after $t^{\prime}$, the applied transport current remains in the DC condition, whilst an AC magnetic field is perpendicularly applied to the wire's surface. Thus, before reaching the first peak of the AC field at the time step ' 3 ', it is possible to observe diverse patterns of evolution for the flux front profile, where a clear competition between the introduced magnetisation currents and the injected transport currents arises. It gives place to a noticeable distortion of the axisymmetric orientation of the original circular flux front profile found at t' (i.e., when $I_{t r}=I_{a}$ and $B_{0 y}=0$ ), until the time step ' 3 ' when $I_{t r}=I_{a}$ and $B_{0 y}=B_{a}$ (see figure 2). In this case, two physical features have been observed from the flux front dynamics of 
profiles of current density. On the one hand, it is possible to state that for an AC field of non dimensional units $b_{a}=B_{a} / B_{P} \leq i_{a}$, being $B_{P}=8\left(\mu_{0} / 4 \pi\right) J_{c} R$ the minimum value of applied magnetic field needed to saturate the magnetic moment of the $\mathrm{SC}$ wire (in absence of transport current) [14, and $i_{a}=I_{a} / I_{c}$ with $I_{c}$ the critical current of the SC wire, slight distortions of the originally circular flux front profile are to be expected during the first cycle of the AC magnetic excitation (see subplots $1-3$ at figure 22. Nevertheless, the position of the centre of the flux free core (region without current) is always shifted away from the geometrical centre of the SC sample; in our case, towards the left side of the $\mathrm{SC}$ given the positive slope of the applied magnetic field, which add positive profiles of magnetization currents at the positive x-quadrants of the SC body. On the other hand, if $b_{a}>i_{a}$, a more acute distortion and displacement of the flux front profile occurs, it due to the added profiles of magnetization current which could eventually lead to the entire disappearance of the flux free core at $b_{a}=1$.

It is worth mentioning that the origin of the deformation of the flux front profile in the case of SC DC wires under AC field conditions, is not caused by the consumption of magnetization currents as it is the case of the $\mathrm{AC}$ wires considered in Ref. 14. It is because of in our case, the current being applied is DC and therefore, the total area occupied by the profiles of current of this nature cannot change, which does not mean that it cannot be redistributed across the cross-section area of the SC sample. Therefore, when the AC field is applied, the occurrence of magnetization currents exerts a Lorentz force which push inwards the distribution of local elements with transport current density $J_{i}=J_{c}$, reducing then the total area of the flux free core until the applied magnetic field is intense enough to saturate the SC sample. As consequence, the Lorentz force added to the system by the incursion of magnetization currents, results in an increase of the local density of power dissipation also known as local energy losses $\mathbf{E} \cdot \mathbf{J}$, what explains why the AC losses in SC samples subjected to AC fields and DC transport currents, can be even greater than the $\mathrm{AC}$ losses in samples subjected to both, AC field and AC transport current, a fact that is presented at the end of this paper.

Continuing with our discussion on the physical characteristics of the distribution of $J_{i}$ profiles in the case of DC SC wires subjected to AC magnetic fields (Figure 1), it can be observed in figure 2 how the first peak-to-peak cycle of the AC field (time steps 3 to 11) cannot be considered for the calculation of the actual AC-losses of the $\mathrm{SC}$ wire, as the size of the flux free core keeps changing during this period of time. In other words, in figure 2 the reader can appreciate in a glance how the $J_{i}$ profiles at the time steps 3 and 11 do not convolute to each other, resulting in a net energy loss that is not accounted if this first peak-to-peak cycle is considered as the foundation for the calculation of the AC-losses. However, if the integral of the local density of power dissipation, $\mathbf{E} \cdot \mathbf{J}$, accounts for the period of time enclosed by the second cycle of the magnetic excitation (time steps 11 to 19), it can be directly seen how the $J_{i}$ distribution across the $\mathrm{SC}$ sample returns to its initial state of magnetization (time step 11), from which the hysteretic losses of the SC sample (per cycle) does not depend any longer on the previous magnetic history, i.e. the dynamics of the flux front profile along the following cycles becomes identical to the one reported between the time steps 11 to 19 . Nevertheless, when $B_{a}$ is as intense as to saturate the SC sample before completing the first peak-to-peak cycle of $B_{0 y}$, we have found that the $\mathrm{AC}$ losses can be accurately calculated from this period of time as no variation on the magnetization loop is observed on the subsequent cycles (see figure 3).

\section{MAGNETIZATION CHARACTERISTICS AND AC-LOSSES}

For the purpose of completeness and with the aim to elucidate if the noticeable long period of magnetic saturation observed for the DC SC wires under transverse AC magnetic field (see figure 3), allow the definition of Boolean-like conditions in the magnetic moment and a unique curve of AC-Losses, both regardless whether the $\mathrm{SC}$ wire is initially magnetized or not, we have performed a similar study to the previous one, but now with $I_{t r}$ being applied after completing a full hysteresis cycle of the $B_{0 y}$ (case 2 in figure 1). Under these circumstances, we have found that when the $\mathrm{SC}$ wire has been premagnetized, the stabilization period of the profiles of current density takes up to two peak-to-peak cycles of the AC field. Nevertheless, once the stabilization period is overcome, the resulting $J_{i}$ profiles and lines of magnetic field for virgin or magnetized samples do not depend on the magnetic history of the SC wire, returning then to the same results displayed in Fig. 2 between the subplots 11 to 19. Thus, regardless of the initial magnetization of the DC SC wire, it is clear that the shape and size of the magnetization loops formed by the AC field (Figure 3), do not change after an initial period of magnetic stabilization which spans for a maximum of two cycles.

A striking feature can be observed from the magnetization loops derived from our experimental conditions. It has to be noticed that as $I_{a}$ increases the shape of the magnetization loop $\left(M_{y}\left(B_{0, y}\right)\right)$ approximate to a squarelike shape (see Fig. 3), reaching the saturation of the magnetic moment at $\mathrm{AC}$ fields of amplitudes lower than $B_{P}$. This phenomenon implies that when the SC wire exhibits the full saturation state, there is the possibility to achieve a fast transition from the magnetically stable region with a positive (or negative) constant magnetic moment, towards a counter magnetic moment but of same amplitude and under well-defined control conditions that might define Boolean-like states in the magnetic moment of DC SC wires subject to transverse AC magnetic fields. In other words, under the right conditions of DC current 




Figure 2. (Color online) Evolution of magnetic flux lines and the corresponding profiles of current density inside the SC wire for the case 1 (see Fig. 1) with $B_{a}=2\left(\mu_{0} / 4 \pi\right) J_{c} R$ and $I_{a}=0.5 I_{c}$. Darker (blue) regions indicates zones with negative elements of current density $J_{i}=-J_{c}$, and the lighter (yellow) regions correspond to $J_{i}=+J_{c}$, otherwise (green) it corresponds to the flux free core where $J_{i}$ and $B_{i}$ are both equal zero. Subplots are labelled accordingly with the monotonic branch of the experimental processes depicted in Fig. 1 .

and AC field, the usefulness of SC wires could also consider the macroscopic transferring of magnetic Boolean data, that theoretically will not interfere with the transmission of transport current, as in ideal conditions the sending and receiving DC current is always the same regardless of the amplitude of $B_{a}$ (neglecting any losses added by the SC joints). Thus, the transmission of magnetic logic data depends on the history of the transverse magnetic excitation $B_{0}$, if proper cryptographic conditions with well-defined Boolean structure can be found.
Nevertheless, the practical realization of this condition can be challenging when $B_{0}$ is needed to be applied just to some specific section of the $\mathrm{SC}$ wire, as the use of a $2 \mathrm{D}$ model implies that the magnetization current loops are closing at the sending and ending terminals of a very long $\mathrm{SC}$ wire, and this situation can be only satisfied if the applied magnetic field is strictly orthonormal to, both the direction of the transport current and the surface of the superconducting wire, otherwise flux cutting effects such as the ones reported in 35, might provoke the closure 


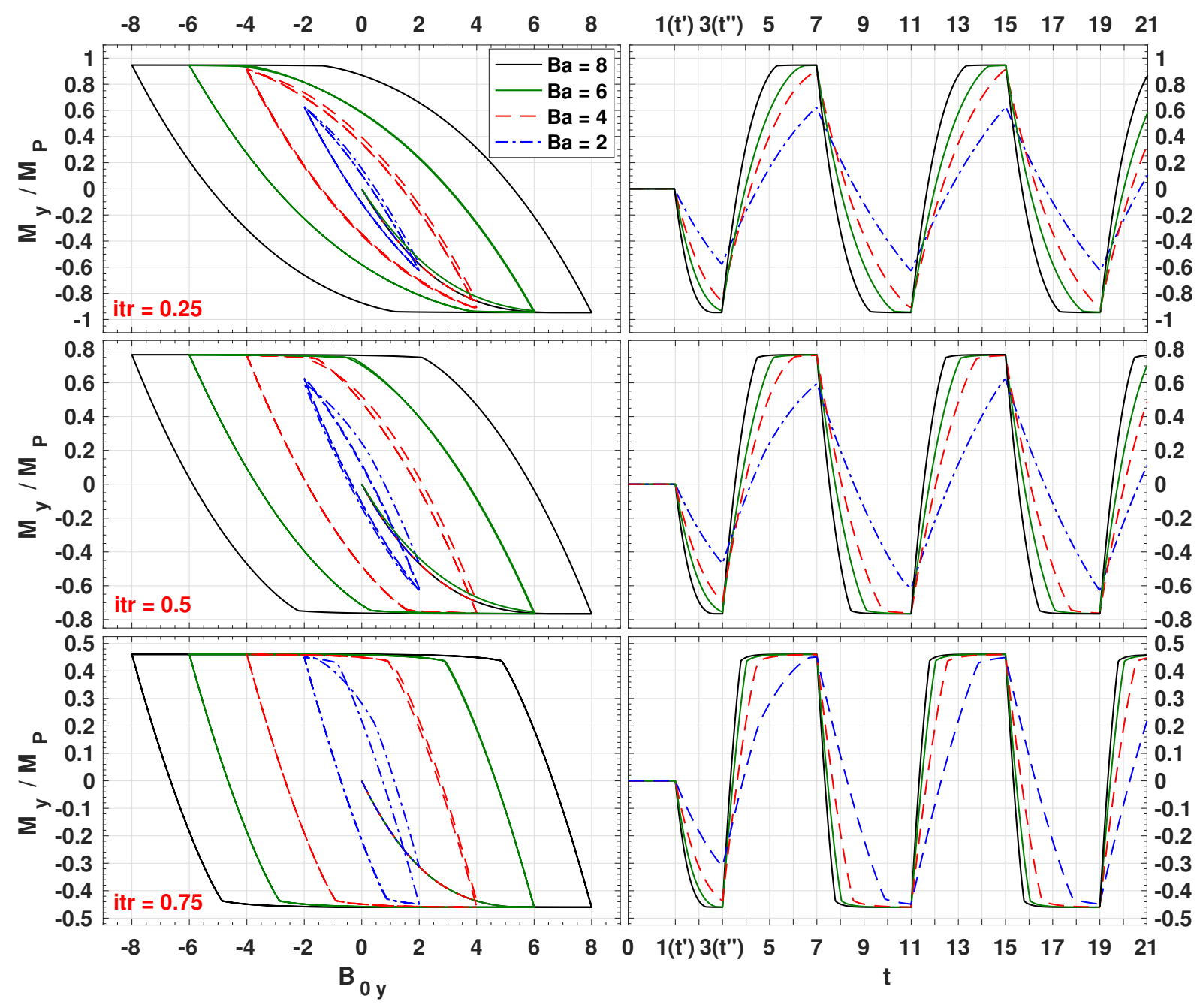

Figure 3. Dimensionless magnetization curves $\left(M_{y} / M_{p}\right)$ as function of the AC field $B_{0 y}$ and the temporal/experimental process of case 1 in Fig. 1. $M_{p}=2 J_{c} R^{3} / 3$ is the analytical solution for the magnetic moment of the sample when it is fully penetrated by a field $B_{P}=8\left(\mu_{0} / 4 \pi\right) J_{c} R$ with zero transport current.

of the magnetization loops before any Boolean-like state could be measured from the transverse magnetization of the SC wire near of the current terminals. However, it is worth mentioning that an experimental proof of these statements is out of scope in this manuscript, but hopefully, by having proven the validness of our statements either by the detailed analysis of the profiles of current density, or the time-dynamics of the magnetization curves, or even by the strict comparison of our numerical results with well acclaimed semi-analytical approaches for the calculation of the AC-losses, this paper will motivate further groups to instigate the experimental realization of magnetization states with the macroscopic Boolean-like properties reported in this manuscript.

However, for showing how a Boolean-like logic can be read from the magnetic moment profile of DC SC wires subjected to AC transverse magnetic field, we must analyse the evolution of the magnetic moment $M_{y}$ (see Fig. 3p) as a function of the time-dependent external magnetic excitation $B_{0 y}(t)$ (see Fig. 1). It is worth reminding that, the resulting magnetization loop for type-II SCs with $I_{t r}<I_{c}$ and $B_{0 y}>0$ under the critical state regime, which is valid at least for frequencies lower than $300 \mathrm{MHz}$ [36], does not depend on the frequency and time-dependence of the function describing the oscillation of the external magnetic field $B_{0 y}(t)$, either it be triangular, sinusoidal, or otherwise, but on its own amplitude $B_{a}$. Nevertheless, the actual value of the magnetic moment at an specific value of applied field, says at a time-step $t$, depends on its own history, i.e, on its current value $B_{0 y}(t)$ and on the sign of its rate of change $\Delta B_{0 y}(t)=B_{0 y}(t)-B_{0 y}(t-1)$. Thus, we have found that for situations where DC transport currents of intensity $I_{a} \geq 0.5 I_{c}$ and transverse applied magnetic fields of amplitudes $B_{a} \geq 6$, in units of $\left(\mu_{0} / 4 \pi\right) J_{c} R$, there are two well defined plateaus where the magnetic moment keeps 
constant at $\mp M_{p^{\prime}}$, with the resulting saturated magnetic moment $\left|M_{p^{\prime}}\right|<|M p|$, being $\left|M_{p}\right|=2 / 3$ the maximum magnetic moment that the SC wire can achieve in the absence of transport current. Surprisingly, these plateaus are observed during the entire period of time from which $B_{0 y}$ ranges from 0 to $\pm B_{a}$, with the signs rule defined by sign of the slope $\Delta B_{0 y}(t)$. To have a clearer picture of this phenomenon, lets take a look to the magnetization curves in Fig. 3 for the case with $I_{a}=0.75 I_{c}$ (bottom pane) and $B_{a} \geq 6$ (solid lines), which are displayed accordingly with the time dynamics of the magnetic excitation shown in Fig. 1. Thus, it has to be noticed that for any applied magnetic field between the time steps 5 and 7, i.e., from $B_{0 y}=0$ to $B_{0 y}=-B_{a}=-6$, the resulting magnetic moment is constant $\left(M_{p^{\prime}} \approx 0.4601\right)$. Then, between the time steps 7 and 9 , i.e., when the sign of $\Delta B_{0 y}(t)$ changes as it goes from $B_{0 y}=-B_{a}$ to $B_{0 y}=0$, the magnetic moment rapidly changes its polarity to $-M_{p^{\prime}}$. Then, if the same analysis is performed for lower intensities of $I_{a}$, we can observe that for $I_{a}=0.5$ (mid-pane of Fig. 3), the same Boolean-like states for the magnetic moment, $M= \pm M_{p^{\prime}}= \pm 0.7661$, is observed between the intervals of applied AC field $B_{0 y}=0$ to $B_{0 y}=\mp B_{a}$ with $B_{a} \geq 6$ (solid lines). Therefore, under these circumstances, we can conclude that for any value of the $\mathrm{AC}$ applied magnetic field with both, negative intensity and negative tendency $\Delta B_{0 y}(t)$, the magnetic moment of the wire keeps at the saturation value $M_{p^{\prime}}$, whilst for a positive intensity of the applied field and a positive tendency $\Delta B_{0 y}(t)$, the magnetic moment of the wire corresponds to the state $-M_{p^{\prime}}$, otherwise, the magnetic moment of the SC wire will be defined between the transient states $\pm M_{p^{\prime}}$, allowing to define a third logic input for $|M| \neq M_{p^{\prime}}$ with the sign of $B_{0 y}$ different to the sign of $\Delta B_{0 y}$, allowing the practical use Boolean-like states in the magnetic moment of type-II superconductors subjected to DC currents and a transversely applied AC magnetic field.

Finally, an ultimate validation of our numerical results has been pursued in Fig. 4. by showing how the calculated AC-losses of the DC superconducting wire under $\mathrm{AC}$ transverse magnetic field, agrees with one of the well known AC-losses formulas of Aleksandr Gurevich,

$$
L\left(B_{\mathrm{a}}, I_{t r}^{d c}\right) \equiv \frac{8 B_{p}^{2}}{3 \mu_{0}}\left\{\begin{array}{l}
b_{\mathrm{a}}^{3}\left(1-\frac{1}{2} b_{\mathrm{a}}\right), \forall \quad b_{\mathrm{a}}<i_{\mathrm{a}}^{\dagger} \\
\text { otherwise } \\
i_{\mathrm{a}}^{\dagger^{3}}\left(1-\frac{1}{2} i_{\mathrm{a}}^{\dagger}\right)+\left(1+i_{\mathrm{a}}^{2}\right)\left(b_{\mathrm{a}}-i_{\mathrm{a}}^{\dagger}\right)
\end{array}\right.
$$

where the dimensionless parameters $i_{a}^{\dagger}=1-i_{a}^{2 / 3}$, with $i_{a}=I_{a} / I_{c}$, and $b_{a}=B_{a} / B_{p}$ have been introduced for the sake of simplicity. This equation combines semianalytical and empirical approaches that at least for high amplitudes of the $\mathrm{AC}$ applied magnetic field and the DC transport current, has shown a remarkable accuracy on the calculation of the AC-losses in contrast with a large set of experimental evidences on NbTi and BSCCO rounded wires [30]. In fact, the Gurevich approaches have

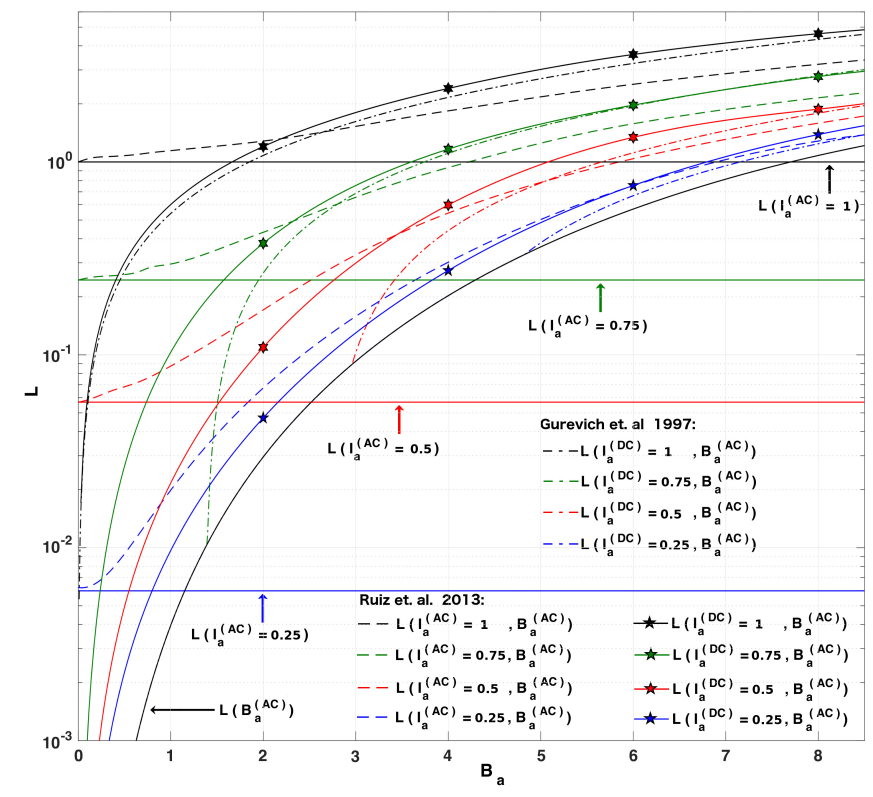

Figure 4. Hysteretic AC losses per cycle of a DC SC wire subjected to a transverse AC magnetic field of amplitude $B_{a}$, and different intensities of the DC transport current $I_{a}=$ $I_{c} / 4, I_{c} / 2,3 I_{c} / 4$, or $I_{c}$, and $L$ units are $\left(\mu_{0} 4 \pi\right) \omega J_{c}^{2} R^{2}$. Our results (solid lines with symbols) are compared with the semiempirical approach of Gurevich et. al. 30] for DC current and AC field (dash-dotted-lines), and with the numerical results of Ruiz et. al. 15] for synchronous AC field and AC current (dashed-lines). The curves of $\mathrm{AC}$ losses for the $\mathrm{SC}$ wire under $\mathrm{AC}$ field and $I_{t r}=0, L\left(B_{a}^{(A C)}\right)$, and AC transport current under no external magnetic field $B_{a}=0, L\left(I_{a}^{(A C)}\right)$, are also shown as lines of reference.

been also shown to be of practical interest on the study of Rutherford-type cables for superconducting particle accelerator magnet coils 37, as well as on a wide number of electric power applications 38, reason why these are considered as a fundamental base to prove the validness of diverse theoretical approaches focused on the reduction of the AC-losses in type-II superconductors [39. However, despite the brilliant simplicity of Gurevich approaches, it is also known that his equations have certain limitations and must be used with caution [14, 30. In this sense, we have found that for the experimental conditions represented in Fig,1, and under the framework of the critical state theory and the formulation introduced in Section 1 of this manuscript, the Gurevich approach, Eq. 5 underestimates the actual contribution of the inductive losses due to the motion of local lines of transport current, as consequence of the magnetic pressure exerted by the $\mathrm{AC}$ field, particularly when $b_{a}<i_{a}^{\dagger}$ (see Fig. 4).

\section{CONCLUDING REMARKS}

For concluding this study, we would like to remind that 
the common interest of studying rounded SC wires, lies on the fact that exact analytical approaches can be found for the calculation of AC losses when isolated excitations are considered, $I_{t r}(t)$ or $B_{0 y}(t)$, both serving as a benchmark for the estimation of the energy losses in practical applications. In fact, it is common to assume that the heat release by a $\mathrm{SC}$ wire under both excitations, $I_{t r}$ and $B_{0 y}$, can be estimated by the linear sum between their individual contributions 16, 40,43, at least for cases with low values of magnetic field and high transport current or vice versa [16, 44, 46]. However, it has been already demonstrated in Ref. 14 that a significant underestimation of the actual AC losses of the system can be obtained if conveniently, existing semi-analytical approaches are invoked when both electromagnetic sources are in AC regime. Thus, we have shown that the AC losses of a DC rounded $\mathrm{SC}$ wire, either premagnetized or not, but subjected to an oscillating transverse magnetic field, cannot be accurately predicted by the well known semi-analytical approaches of Gurevich et al. [30, under all possible combinations of magnetic field and transport current. Thus, in this paper we have demonstrated that for high values of magnetic field $B_{a} \geq B_{P} / 2=4$, in units of $\left(\mu_{0} / 4 \pi\right) J_{c} R$, the attained values of the AC losses are nearly the same than those obtained in Ref. 14 for the case of AC field and AC transport current, as well as with the semi-analytical approach introduced by Gurevich et al. through Eq. 5 Nevertheless, for low values of magnetic field $B_{a}<B_{P} / 2$, the semi-analytical approach is proven to underestimate the actual contribution of the inductive losses due to the motion of local lines of transport current, particularly when $b_{a}<i_{a}^{\dagger}$, with $i_{a}^{\dagger}=1-i_{a}^{2 / 3}$. A further remarkable feature can be observed in Fig. 4. as regardless of the type of $I_{t r}$ considered, either DC or $\mathrm{AC}$, when the $\mathrm{SC}$ wire is subjected to a transverse $\mathrm{AC}$ magnetic field, the resulting $\mathrm{AC}$ losses are nearly equal as long as the condition $i_{a}^{*}=1-i_{a} \leq b_{a}$ is satisfied, therefore defining the limit from which the technological advantages of using $\mathrm{SC}$ wires for $\mathrm{DC}$ applications sharing the right of way with $\mathrm{AC}$ lines, competes with the case of using the $\mathrm{SC}$ wire for the transmission of $\mathrm{AC}$ current. Finally, it is worth mentioning that despite the theoretical soundness of our results, an experimental prove of the usefulness or viability of the predicted macroscopic Boolean states in the magnetic moment of DC superconducting wires is still to be confirmed. However, our study hopes to motivate groups of experimentalist to demonstrate the applicability of our results beyond the theoretical limits imposed by the 2D geometry of our case of study, and the implications of the critical state approach.

\section{ACKNOWLEDGEMENT}

We would like to thank A. Badía-Majós and Y. Genenko for their valuable comments and discussion. This work was supported by the High Performance Computing Cluster (ALICE) at the University of Leicester. B. Robert thanks the Scholarship unit of the Niger Delta Development Commission for their financial support.

\section{REFERENCES}

[1] Z. Zhang, S. Venuturumilli, M. Zhang, and W. Yuan, Superconducting Cables - Network feasibility study work package 1 , Innovation, Next Generation Networks (Western Power Distribution, 2016) p. 89.

[2] H. Thomas, A. Marian, A. Chervyakov, S. St'uckrad, D. Salmieri, and C. Rubbia, Renewable and Sustainable Energy Reviews 55, 59 (2016).

[3] X. Zhang, Z. Zhong, H. S. Ruiz, J. Geng, and T. A. Coombs, Superconductor Science and Technology 30, 025010 (2017)

[4] H. S. Ruiz, X. Zhang, and T. A. Coombs, IEEE Transactions on Applied Superconductivity 25, 5601405 (2015).

[5] C. P. Bean, Rev. Mod. Phys. 36, 31 (1964).

[6] A. Badía-Majós, C. López, and H. S. Ruiz, Phys. Rev. B 80, 144509 (2009)

[7] H. S. Ruiz and A. Badía-Majós, Superconductor Science and Technology 23, 105007 (2010)

[8] G. P. Mikitik, Y. Mawatari, A. T. S. Wan, and F. Sirois, IEEE Transactions on Applied Superconductivity 23, 8001920 (2013)

[9] M. Baghdadi, H. S. Ruiz, and T. A. Coombs, Applied Physics Letters 104, 232602 (2014).
[10] A. Campbell, M. Baghdadi, A. Patel, D. Zhou, K. Y. Huang, Y. Shi, and T. Coombs, Superconductor Science and Technology 30, 034005 (2017)

[11] F. Liang, T. Qu, Z. Zhang, J. Sheng, W. Yuan, Y. Iwasa, and M. Zhang, Superconductor Science and Technology 30, 094006 (2017).

[12] S. Celebi, F. Sirois, and C. Lacroix, Superconductor Science and Technology 28, 025012 (2015).

[13] J. L. Giordano, J. Luzuriaga, A. Badía-Majós, G. Nieva, and I. Ruíz-Tagle, Superconductor Science and Technology 19, 385 (2006)

[14] H. S. Ruiz, A. Badía-Majós, Y. A. Genenko, H. Rauh, and S. V. Yampolskii, Applied Physics Letters 100, $112602(2012)$

[15] H. S. Ruiz and A. Badía-Majós, Journal of Applied Physics 113, 193906 (2013).

[16] J. Ogawa, S. Fukui, T. Oka, T. Sato, H. Kojima, M. Shibayama, and M. Egawa, IEEE Transactions on Applied Superconductivity 21, 3325 (2011)

[17] F. Grilli, E. Pardo, A. Stenvall, D. N. Nguyen, W. Yuan, and F. Gömöry, IEEE Transactions on Applied Superconductivity 24, 78 (2014) 
[18] A. Stenvall, V. Lahtinen, and M. Lyly, Superconductor Science and Technology 27, 104004 (2014)

[19] J. Borwein and A. Lewis, Convex Analysis and Nonlinear Optimization, theory and examples (Springer-Verlag, New York, 2006) p. 316.

[20] A. J. Kurdila and M. Zabarankin, Convex Functional Analysis (Birkhauser Verlag, 2005) p. 238.

[21] Y. Zhu and A. Cangellaris, Multigrid finite element methods for electromagnetic field modeling (Wiley-IEEE, 2006) p. 408.

[22] A. Conn, G. Gould, and P. Toint, Lancelot: A Fortran Package for Large-Scale Nonlinear Optimization (Release A) (Springer-Verlag Berlin Heidelberg, 1992) p. 332.

[23] A. R. Conn, N. I. M. Gould, and P. Toint, SIAM Journal on Numerical Analysis 28, 545 (1991)

[24] F. Gömöry, R. Tebano, A. Sanchez, E. Pardo, C. Navau, I. Husek, F. Strycek, and P. Kovac, Superconductor Science and Technology 15, 1311 (2002).

[25] F. Sirois and F. Grilli, Superconductor Science and Technology 28, 043002 (2015)

[26] A. M. Campbell, Superconductor Science and Technology 22, 034005 (2009).

[27] F. Gömöry, M. Vojenčiak, E. Pardo, M. Solovyov, and J. Šouc, Superconductor Science and Technology 23, $034012(2010)$

[28] C. P. Bean, Journal of Applied Physics 41, 2482 (1970), http://dx.doi.org/10.1063/1.1659249.

[29] W. J. Carr, AC loss and macroscopic theory of superconductors (Taylor \& Francis, 2001) p. 224.

[30] A. V. Gurevich, R. G. Mints, and A. L. Rakhmanov, Physics of Composite Superconductors (Begell House, New York, 1997) p. 348.

[31] N. Amemiya and Y. Ohta, Physica C: Superconductivity 357, 1134 (2001)

[32] N. Nibbio and S. Stavrev, IEEE Transactions on Applied Superconductivity 11, 2627 (2001)
[33] V. Lahtinen, M. Lyly, A. Stenvall, and T. Tarhasaari, $\mathrm{Su}-$ perconductor Science and Technology 25, 115001 (2012).

[34] S. T. Ranecky, H. Watanabe, J. Ogawa, T. Oka, D. G'olden, L. Alff, and Y. A. Genenko, Superconductor Science and Technology 30, 085005 (2017)

[35] H. S. Ruiz, C. López, and A. Badía-Majós, Phys. Rev. B 83, 014506 (2011)

[36] L. E. Svistov and S. A. Vitkalov, Czechoslovak Journal of Physics 46, 1267 (1996).

[37] A. Devred, Superconducting magnets for particle accelerators and storage rings (Wiley Encyclopedia of Electrical and Electronic Engineering, 1999) p. 332.

[38] D. Larbalestier, A. Gurevich, D. M. Feldmann, and A. Polyanskii, Nature 414, 368 (2001).

[39] D. Abraimov, A. Gurevich, A. Polyanskii, X. Y. Cai, A. Xu, S. Pamidi, D. Larbalestier, and C. L. H. Thieme, Superconductor Science and Technology 21, 082004 (2008)

[40] S. Zannella, L. Montelatici, G. Grenci, M. Pojer, L. Jansak, M. Majoros, G. Coletta, R. Mele, R. Tebano, and F. Zanovello, IEEE Transactions on Applied Superconductivity 11, 2441 (2001)

[41] H. Tonsho, S. Fukui, T. Sato, M. Yamaguchi, S. Torii, T. Takao, and K. Ueda, IEEE Transactions on Applied Superconductivity 13, 2368 (2003)

[42] J. Ogawa, M. Shiokawa, M. Ciszek, and O. Tsukamoto, IEEE Transactions on Applied Superconductivity 13, 1735 (2003).

[43] M. Vojenčiak, J. Šouc, J. M. Ceballos, F. Gömöry, B. Klinčok, E. Pardo, and F. Grilli, Superconductor Science and Technology 19, 397 (2006).

[44] R. Inada, K. Tateyama, Y. Nakamura, A. Oota, C. Li, and P. Zhang, Superconductor Science and Technology 20, 138 (2007).

[45] Z. Jiang, N. Amemiya, N. Ayai, and K. Hayashi, Superconductor Science and Technology 17, 1311 (2004)

[46] J. J. Rabbers, B. ten Haken, and H. H. J. ten Kate, Review of Scientific Instruments 72, 2365 (2001). 